\title{
Jost function for coupled channels *
}

\author{
S. A. Rakityansky ${ }^{\dagger}$ and S. A. Sofianos ${ }^{\ddagger}$ \\ Department of Physics, University of South Africa, P.O.Box 392, Pretoria 0003, South Africa
}

Almost any textbook on the scattering theory has a chapter devoted to the Jost function, but none of them gives a practical recipe for its calculation. Thus one usually gets a feeling that the Jost function is a pure mathematical object, elegant and useful in formal theory, but impractical in computations. This is even more so in the case of multi-channel problems, where the Jost function is, in fact, a matrix. Recently, we proposed an exact method for direct calculation of the Jost function for central potentials (which may have Coulombic tails) [1,2] and the Jost matrix for non-central short range potentials [3]. This method works for all real or complex momenta of physical interest, including the spectral points corresponding to bound and Siegert states. In this work we extend it for potentials which couple channels with different thresholds.

There are three different types of physical problems associated with the Schrödinger equation: bound, scattering, and resonance state problems. They differ in the boundary conditions imposed on the wave function at large distances. Alternatively, a solution can be specified by the boundary conditions at the origin. For example, in the single-channel case the solution which vanishes as $r \rightarrow 0$ exactly like the Riccati-Bessel function, is called the regular solution. It is well known that if the potential couples $N$ channels, the Schrödinger equation

$$
\left[\partial_{r}^{2}+2 \mu_{n}\left(E-E_{n}\right)-\frac{\ell(\ell+1)}{r^{2}}\right] u_{n}(E, r)=\sum_{n^{\prime}=1}^{N} V_{n n^{\prime}}(r) u_{n^{\prime}}(E, r)
$$

has $2 N$ linearly independent column-solutions

$$
\left(\begin{array}{c}
u_{1} \\
u_{2} \\
\vdots \\
u_{N}
\end{array}\right)_{1},\left(\begin{array}{c}
u_{1} \\
u_{2} \\
\vdots \\
u_{N}
\end{array}\right)_{2},\left(\begin{array}{c}
u_{1} \\
u_{2} \\
\vdots \\
u_{N}
\end{array}\right)_{3}, \cdots\left(\begin{array}{c}
u_{1} \\
u_{2} \\
\vdots \\
u_{N}
\end{array}\right)_{2 N}
$$

and only half of them are regular at the point $r=0$. We can therefore combine all the

*talk given at XVI European Conference on Few-Body Problems in Physics, Autrans, June 1998. To appear in Few Body Systems Suppl.

${ }^{\dagger}$ E-mail address: rakitsa@kiaat.unisa.ac.za

${ }^{\ddagger}$ E-mail address: sofiasa@kiaat.unisa.ac.za 
regular solutions (to distinguish them we use the notation $\phi$ ) in a square matrix,

$$
\left(\begin{array}{c}
\phi_{1} \\
\phi_{2} \\
\vdots \\
\phi_{N}
\end{array}\right)_{1} \oplus\left(\begin{array}{c}
\phi_{1} \\
\phi_{2} \\
\vdots \\
\phi_{N}
\end{array}\right)_{2} \oplus \cdots \Longrightarrow\left(\begin{array}{cccc}
\phi_{11} & \phi_{12} & \cdots & \phi_{1 N} \\
\phi_{21} & \phi_{22} & \cdots & \phi_{2 N} \\
\vdots & \vdots & \ddots & \vdots \\
\phi_{N 1} & \phi_{N 2} & \cdots & \phi_{N N}
\end{array}\right) \equiv\|\phi(E, r)\|,
$$

which obeys the (matrix) Schrödinger equation with the boundary condition

$$
\lim _{r \rightarrow 0} \phi_{n n^{\prime}}(E, r) / j_{\ell}\left(k_{n^{\prime}} r\right)=\delta_{n n^{\prime}}
$$

where $j_{\ell}(z)$ is the Riccati-Bessel function and $k_{n}=\sqrt{2 \mu_{n}\left(E-E_{n}\right)}$ is the channel momentum. Any physical solution must be regular at the origin and therefore it is a linear combination of the regular columns. In this respect the matrix $\|\phi\|$ can be called the regular basis.

In order to obtain this basis in the form which is most suitable for describing its long-range asymptotics, we replace the unknown matrix-function $\|\phi(E, r)\|$ by two others, $\left\|\mathcal{F}^{(-)}(E, r)\right\|$ and $\left\|\mathcal{F}^{(+)}(E, r)\right\|$, via the following ansatz

$$
\phi_{n n^{\prime}}(E, r)=\frac{1}{2}\left[h_{\ell}^{(-)}\left(k_{n} r\right) \mathcal{F}_{n n^{\prime}}^{(-)}(E, r)+h_{\ell}^{(+)}\left(k_{n} r\right) \mathcal{F}_{n n^{\prime}}^{(+)}(E, r)\right],
$$

with the additional constraint conditions

$$
h_{\ell}^{(-)}\left(k_{n} r\right) \partial_{r} \mathcal{F}_{n n^{\prime}}^{(-)}(E, r)+h_{\ell}^{(+)}\left(k_{n} r\right) \partial_{r} \mathcal{F}_{n n^{\prime}}^{(+)}(E, r)=0
$$

which make the derivative of the wave functions continuous even if the potential has a sharp cut-off. Substituting this ansatz into the Schrödinger equation we derive the following system of first order differential equations for these new unknown matrices,

$$
\partial_{r} \mathcal{F}_{n n^{\prime}}^{(\mp)}=\mp \frac{1}{2 i k_{n}} h_{\ell}^{( \pm)}\left(k_{n} r\right) \sum_{n^{\prime \prime}} V_{n n^{\prime \prime}}(r)\left[h_{\ell}^{(+)}\left(k_{n^{\prime \prime}} r\right) \mathcal{F}_{n^{\prime \prime} n^{\prime}}^{(+)}+h_{\ell}^{(-)}\left(k_{n^{\prime \prime}} r\right) \mathcal{F}_{n^{\prime \prime} n^{\prime}}^{(-)}\right]
$$

The boundary conditions corresponding to (2) are

$$
\lim _{r \rightarrow 0} \mathcal{F}_{n n^{\prime}}^{( \pm)}(E, r)=\delta_{n n^{\prime}}
$$

Explicit inclusion of the Riccati-Hankel functions into the basis (3) guarantees the correct asymptotic behaviour of the physical solution.

In constructing a physical wave function $\varphi_{n}(E, r)$ one requires the appropriate coefficients $c_{n}$ in the linear combination of the regular columns,

$$
\varphi_{n}(E, r)=\sum_{n^{\prime}} \phi_{n n^{\prime}}(E, r) c_{n^{\prime}}
$$

For bound and resonant states, for example, in the physical wave function only the term proportional to $h^{(+)}$survives at large distances. This gives the homogeneous system of equations

$$
\sum_{n^{\prime}} \mathcal{F}_{n n^{\prime}}^{(-)}(E, \infty) c_{n^{\prime}}=0
$$


which has a non-trivial solution if and only if its determinant is zero, i. e.,

$$
\operatorname{det}\left\|\mathcal{F}^{(-)}(E, \infty)\right\|=0 \text {. }
$$

It is easily seen that this is the determinant of the Jost matrix $\|F(E)\|$ which is defined by the asymptotic behaviour of the regular basis [4]

$$
\phi_{n n^{\prime}}(E, r) \underset{r \rightarrow \infty}{\longrightarrow} \frac{1}{2}\left[h_{\ell}^{(-)}\left(k_{n} r\right) F_{n n^{\prime}}(E)+h_{\ell}^{(+)}\left(k_{n} r\right) \tilde{F}_{n n^{\prime}}(E)\right] .
$$

We can calculate this matrix by solving the differential equations (5) from $r=0$ up to a large radius where the potential is insignificant. Since the potential vanishes, the right hand sides of Eqs.(5) disappear at large distances. Hence the derivatives $\partial_{r}\left\|\mathcal{F}^{(\mp)}(E, r)\right\|$ become zero which means that asymptotically the matrix-functions $\left\|\mathcal{F}^{(\mp)}(E, r)\right\|$ become constants coinciding with $\|F(E)\|$ and $\|\tilde{F}(E)\|$ respectively.

Similarly to the single-channel case (see Refs. [1 [3]) the above scheme can be easily implemented only for bound and scattering states. In the resonance domain of the complex energy plane the Riccati-Hankel functions $h_{\ell}^{(+)}\left(k_{n} r\right)$, and therefore the derivatives of $\left\|\mathcal{F}^{(-)}(E, r)\right\|$ at large distances, become infinitely large which means that the limit $\left\|\mathcal{F}^{(-)}(E, \infty)\right\|$ does not exist. Thus in order to calculate the Jost matrix in this domain we need to perform a complex rotation of the independent variable $r, r=x \exp (i \theta)$, in Eqs.(5), where $x \geq 0$ and $0 \leq \theta<\pi / 2$. This results in a movement of the unitary cuts at each threshold downwards as is shown in Fig. 1. After such a rotation $h_{\ell}^{(+)}\left(k_{n} r\right)$ vanishes in all areas above the turned cuts, which guarantees the existence of the limit $\left\|\mathcal{F}^{(-)}(E, \infty)\right\|$ there.

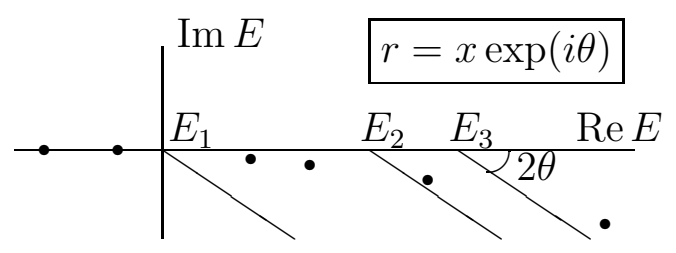

Figure 1. Complex rotation of the coordinate by an angle $\theta$ results in a rotation of the unitary cuts by $2 \theta$.

By locating the zeros of the Jost-matrix determinant we can find the resonance energies and the total widths. The determination of the partial widths, however, is a difficult task for most methods used in the past. In our approach this is not a problem at all. Indeed, knowing the Jost matrix, we can easily obtain the $S$-matrix at a given complex energy as

$$
\|S(E)\|=\left\|\mathcal{F}^{(+)}(E, \infty)\right\| \cdot\left\|\mathcal{F}^{(-)}(E, \infty)\right\|^{-1}
$$

which, generally, consists of two terms, viz.

$$
S_{n n^{\prime}}(E)=S_{n n^{\prime}}^{\mathrm{bg}}-i \frac{\sqrt{\Gamma_{n} \Gamma_{n^{\prime}}}}{E-E_{r}+i \Gamma / 2} .
$$

One of them is the smooth background term $S_{n n^{\prime}}^{\mathrm{bg}}$ and the other a singular resonance term. Thus a partial width is the limit

$$
\Gamma_{n}=\left|\lim _{E \rightarrow\left(E_{r}-i \Gamma / 2\right)}\left(E-E_{r}+i \Gamma / 2\right) S_{n n}(E)\right| .
$$


Practically, to find such a limit we calculate the $S$-matrix at a point very close to the resonant energy and multiply it by the corresponding energy difference.

To demonstrate the ability of the method we consider a numerical example proposed by Noro and Taylor in Ref. [5] where they used the $S$-wave potential

$$
\|V(r)\|=\left(\begin{array}{rr}
-1.0 & -7.5 \\
-7.5 & 7.5
\end{array}\right) r^{2} e^{-r}
$$

which describes a two-channel system with the thresholds $E_{1}=0$ and $E_{2}=0.1$ (atomic units). By expanding the resonance wave function in a series of square-integrable functions, Noro and Taylor located the first resonance generated by this potential. We reproduce their result and found also three bound states and several broad resonances. For comparison we put the parameters of the first resonance in Table 1. The digits shown do not change when the rotation angle is changed. This demonstrates the accuracy of our method.

\begin{tabular}{|c|c|c|}
\hline & our results & Noro \& Taylor \\
\hline$E_{r}$ & 4.7681968188 & 4.7682 \\
$\Gamma$ & 0.0014201920 & 0.001420 \\
$\Gamma_{1}$ & 0.000051103 & 0.000059 \\
$\Gamma_{2}$ & 0.001368733 & 0.001361 \\
\hline
\end{tabular}

Table 1.

Values are given in atomic units

In conclusion, we propose a method based on simple differential equations of the first order, which can be easily solved numerically. Thus, the spectrum generated by any given potential can be thoroughly investigated. At the same time the physical wave function can be obtained having the correct asymptotic behaviour. 


\section{REFERENCES}

[1] S. A. Rakityansky, S. A. Sofianos, K. Amos, Nuovo Cim., B111 (1996) 363

[2] S. A. Sofianos, S. A. Rakityansky, Journal of Phys. A30 (1997) 3725

[3] S. A. Rakityansky, S. A. Sofianos, Journal of Phys. A31 (1998) 5149

[4] J. R. Taylor: Scattering Theory. New York: John Wiley \& Sons.

[5] T. Noro, H. S. Taylor, J. Phys., B13 (1980) L377 